\section{Perfil das intoxicações em adolescentes no Brasil no período de 1999 a 2001}

\author{
Profile of poisonings among Brazilian \\ adolescents from 1999 to 2001
}

Rosany Bochner 1

\section{Introdução}

This paper analyzes the profile of poisonings among adolescents as reported by the Brazilian National Poisoning Information System (SINITOX) database. This database covers cases and deaths secondary to human poisoning recorded by Poison Control Centers (1999-2001). With relation to cases, venomous animals (33.7\%) and medicines (25.7\%) are the main toxic agents in pre-adolescents (10-14 years). For adolescents (15-19 years), medicines ranked first (33.0\%), followed by bites/stings from venomous animals (21.4\%). There were 35 deaths among pre-adolescents, $54.3 \%$ resulting from accidental poisoning, $54.3 \%$ in males, and $31.4 \%$ caused by venomous animals. Ninety-eight deaths were reported among adolescents, $72.4 \%$ of which were suicides, $54.1 \%$ in females, and $38.8 \%$ resulting from pesticide poisoning.

Poisoning; Toxic Substances; Adolescent
Segundo o Censo Demográfico 2000, adolescentes de 10 a 19 anos representam a maior parcela da população brasileira. São mais de 35 milhões, o que corresponde à cerca de $21 \%$ da população total do país recenseada no ano 2000 em 169.799.170 habitantes 1.

A adolescência é um período que se situa entre a infância e a maturidade, que se caracteriza por profundas modificações de ordem fisiológica, psicológica e social, e outros aspectos relevantes que se distinguem das de outros períodos da vida humana 2,3 .

A adolescência é uma fase de novas sensações e experiências antes completamente desconhecidas. É por esta razão que é considerada o período, por excelência, de risco com relação ao uso de substâncias psicoativas e danos eventualmente associados a este consumo 4,5 .

O Sistema Nacional de Informações TóxicoFarmacológicas (SINITOX), vinculado à Fundação Oswaldo Cruz (FIOCRUZ), tem como principal atribuição coordenar o processo de coleta, compilação, análise e divulgação dos casos de intoxicação e envenenamento registrados no país pelos Centros de Informação e Assistência Toxicológica localizados em 17 Estados brasileiros 6,7,8. No período de 1999 a 2001, o SINITOX registrou 218.692 casos de intoxicação humana, que resultaram em 1.233 óbitos. Adolescentes de 10 a 19 anos responderam por 
$15,5 \%$ dos casos e $10,8 \%$ dos óbitos, representando a terceira faixa etária mais atingida com relação ao registro de casos e a quinta quanto aos óbitos 9,10,11.

O estudo desse universo de 33.901 casos e 133 óbitos impõe uma análise mais aprofundada, visando delinear o perfil das intoxicações em adolescentes no Brasil.

\section{Metodologia}

\section{Definição das faixas etárias a serem estudadas}

Contrariamente às impressões populares de que a adolescência constitui um período claramente demarcado, há muita disparidade de pontos de vista quanto ao seu início e término. Alguns autores admitem limites aproximados para a adolescência. Com relação ao seu início, alguns consideram dez anos e meio, outros 12 anos e outros ainda fazem distinção ao sexo, 13 anos para meninas e 14 para meninos. A definição de seu término também apresenta variações, 18 anos para uns, 21 ou 25 para outros 12 .

Cada cultura possui um conceito de adolescência, baseando-se sempre nas diferentes idades para definir esse período. No Brasil, o Estatuto da Criança e do Adolescente considera criança, para os efeitos da lei, a pessoa até doze anos incompletos, e adolescente, aquele entre 12 e 18 anos de idade 13 .

A Organização Pan-Americana da Saúde (OPAS) considera a adolescência como um processo primariamente biológico, que abrange o período de 10 a 19 anos e compreende a préadolescência (10 a 14 anos) e a adolescência propriamente dita (15 a 19 anos) e, considera a juventude como uma categoria fundamentalmente sociológica, que se estende dos 15 aos 24 anos de idade e compreende também duas faixas distintas: 15 a 19 e 20 a 24 anos 14 .

As faixas etárias consideradas pelo SINITOX $(<1,1-4,5-9,10-14,15-19,20-29,30-39,40-49$, 50-59, 60-69, 70-79 e $\geq 80$ ) 9,10,11, impõem limites ao estudo no sentido que inviabiliza a análise do comportamento das intoxicações em qualquer outra faixa etária, o que impede a realização de comparações diretas entre os dados do SINITOX com resultados apresentados em artigos que fazem uso de outras faixas etárias.

Como a OPAS considera duas faixas etárias, estas contempladas pelo SINITOX, para classificar os adolescentes em dois grupos distintos, acreditamos ser interessante analisar o comportamento das intoxicações em pré-adoles- centes de 10 a 14 anos e em adolescentes de 15 a 19 anos separadamente.

\section{Procedimento de análise}

Foi realizada uma análise descritiva da base de dados do SINITOX referente ao período de 1999 a 2001. Esta base é composta de tabelas, e não de registros individuais, provenientes de 31 Centros de Informação e Assistência Toxicológica localizados em 17 Estados do país. É importante ressaltar que iremos analisar a totalidade dos casos registrados pelo SINITOX para o Brasil no período de 1999 a 2001, o que é diferente da totalidade dos casos ocorridos no país nesse mesmo período, porque, além do número de centros ser insuficiente para cobrir toda a extensão territorial do país, a notificação dos casos a esses centros é espontânea, sendo realizada pela própria vítima ou seus familiares com o objetivo de obter informação sobre como proceder e onde buscar atendimento, bem como por profissionais de saúde que buscam informações sobre o tratamento a ser realizado 7,8,9,10,11.

Os casos registrados pelos centros são consolidados em seis tabelas, que relacionam sempre o agente tóxico com uma das seguintes seis variáveis: vítima, circunstância, faixa etária, sexo, zona de ocorrência e evolução. Já os óbitos são registrados por meio de uma tabela que fornece informações detalhadas para cada um deles acerca do agente tóxico, circunstância, faixa etária e sexo.

Essas características do sistema limitaram nossa análise aos casos de intoxicação humana distribuídos por faixa etária e ao detalhamento dos óbitos por agente tóxico, circunstância, faixa etária e sexo, para cada uma das regiões geográficas do país. A análise das variáveis circunstância e sexo foi realizada com base em todos os casos, independente da faixa etária.

Dentre os agentes tóxicos considerados pelo SINITOX, temos os produtos químicos industriais, que incluem: derivados de petróleo, inalantes, tintas e vernizes, colas e adesivos, álcool, gases, monóxido de carbono, cola de sapateiro. Os solventes ou inalantes, utilizados como drogas de abuso, integram este conjunto e merecem uma atenção especial ao analisar o perfil das intoxicações em adolescentes. Uma maneira de distinguir os produtos usados como drogas dos demais, seria considerar as intoxicações por produtos químicos industriais associadas com a circunstância "abuso". No entanto, mais uma vez, a estrutura da base de dados do SINITOX não permite este procedimento para cada uma das faixas etárias, limitando 
a análise à circunstância e ao agente tóxico de todas as intoxicações, sem distinção de idade.

\section{Cálculo do coeficiente de incidência}

Para se proceder ao cálculo dos coeficientes de incidência das intoxicações registradas pelo SINITOX no período de 1999 a 2001, distribuídos por faixa etária, consideramos a população do país recenseada em 2000 1, uma vez que este ano é o ponto médio do intervalo considerado, e subtraímos as populações dos Estados e do Distrito Federal que não possuíam centros neste período (Rondônia, Acre, Roraima, Amapá, Tocantins, Maranhão, Piauí, Alagoas e Sergipe).

Os coeficientes são obtidos dividindo o total de casos registrados no período, segundo agente tóxico e faixa etária, pela população ajustada, como explicado acima, multiplicada por três, com exceção dos Estados de Pernambuco e Mato Grosso do Sul que tiveram suas populações multiplicadas por dois, pois estes estados não tiveram seus dados referentes ao ano de 2002 registrados pelo SINITOX.

\section{Resultados}

Os três principais agentes tóxicos envolvidos nas intoxicações de adolescentes de 15 a 19 anos foram: medicamentos (33\%), animais peçonhentos $(21,4 \%)$ e raticidas $(8,5 \%)$. Com relação aos pré-adolescentes de 10 a 14 anos, prevaleceram os animais peçonhentos $(33,7 \%)$, seguidos dos medicamentos $(25,7 \%)$ e dos animais não-peçonhentos (7,3\%) (Tabela 1$)$.

Para os adolescentes, predominaram os animais peçonhentos seguidos dos medicamentos nas regiões Norte, Nordeste e Centrooeste. Já nas regiões Sudeste e Sul ocorreu exatamente o inverso: os medicamentos corresponderam a $41 \%$ e $32,4 \%$ dos registros, respectivamente. Considerando os pré-adolescentes, os animais peçonhentos, seguidos dos medicamentos foram também os principais agentes tóxicos nas regiões Nordeste, Sul e Centrooeste. Na região Norte predominaram os animais peçonhentos seguidos dos animais nãopeçonhentos e na região Sudeste, os medicamentos são seguidos dos animais peçonhentos (Tabela 1).

\begin{tabular}{|c|c|c|c|c|c|c|c|c|c|c|c|c|}
\hline \multirow[t]{3}{*}{ Agentes tóxicos } & \multirow{2}{*}{\multicolumn{5}{|c|}{ Pré-adolescentes de 10 a 14 anos }} & \multicolumn{7}{|c|}{ Adolescentes de 15 a 19 anos } \\
\hline & & & & & & Brasil & & & Regiões & & & Brasil \\
\hline & Norte & Nordeste & Sudeste & Sul & $\begin{array}{l}\text { Centro- } \\
\text { oeste }\end{array}$ & & Norte & Nordeste & Sudeste & Sul & $\begin{array}{l}\text { Centro- } \\
\text { oeste }\end{array}$ & \\
\hline Medicamentos & 9,5 & 12,1 & 36,4 & 22,8 & 15,3 & 25,7 & 16,6 & 21,4 & 41,0 & 32,4 & 20,6 & 33,0 \\
\hline Agrotóxicos de uso agrícola & 4,1 & 1,7 & 4,2 & 4,9 & 1,9 & 3,7 & 7,2 & 3,9 & 7,6 & 8,6 & 5,6 & 6,9 \\
\hline Agrotóxicos de uso doméstico & 1,4 & 1,5 & 3,5 & 2,2 & 3,0 & 2,7 & 2,0 & 2,5 & 4,0 & 2,6 & 5,2 & 3,4 \\
\hline Produtos veterinários & - & 0,7 & 0,6 & 0,8 & 1,9 & 0,8 & 0,3 & 0,7 & 0,7 & 1,3 & 1,2 & 0,9 \\
\hline Raticidas & 7,2 & 9,9 & 4,6 & 1,9 & 2,1 & 4,8 & 13,0 & 17,6 & 6,7 & 5,1 & 3,3 & 8,5 \\
\hline Domissanitários & 1,8 & 2,6 & 7,6 & 4,3 & 2,7 & 5,2 & 3,6 & 3,8 & 6,4 & 5,0 & 4,9 & 5,3 \\
\hline Cosméticos & 0,9 & 0,3 & 0,7 & 0,5 & 0,3 & 0,5 & 1,0 & 0,3 & 0,4 & 0,6 & 0,3 & 0,4 \\
\hline Produtos químicos industriais & 2,7 & 2,8 & 5,3 & 4,2 & 3,9 & 4,3 & 3,6 & 2,6 & 5,4 & 5,2 & 4,4 & 4,7 \\
\hline Metais & - & 0,2 & 0,2 & 0,5 & 0,1 & 0,3 & - & 0,1 & 0,2 & 0,4 & 0,1 & 0,2 \\
\hline Drogas de abuso & - & 1,9 & 3,6 & 1,3 & 1,3 & 2,4 & 2,9 & 1,9 & 6,5 & 5,0 & 2,0 & 4,8 \\
\hline Plantas & 1,8 & 2,1 & 3,2 & 2,7 & 3,8 & 2,9 & 0,7 & 1,5 & 1,3 & 1,4 & 1,6 & 1,4 \\
\hline Alimentos & 18,0 & 1,3 & 0,9 & 0,3 & 6,9 & 1,6 & 14,0 & 0,7 & 0,8 & 0,3 & 2,6 & 1,0 \\
\hline Animais peçonhentos & 28,8 & 48,9 & 18,2 & 42,6 & 51,4 & 33,7 & 22,1 & 32,1 & 11,3 & 24,6 & 44,0 & 21,4 \\
\hline Animais não-peçonhentos & 19,8 & 10,8 & 5,5 & 8,2 & 2,3 & 7,3 & 8,5 & 8,6 & 3,7 & 5,8 & 2,0 & 5,2 \\
\hline Desconhecido & 1,8 & 2,5 & 4,3 & 1,9 & 2,8 & 3,1 & 1,6 & 1,8 & 3,1 & 1,2 & 1,7 & 2,2 \\
\hline Outro & 2,3 & 0,7 & 1,4 & 1,0 & 0,4 & 1,1 & 2,9 & 0,6 & 0,8 & 0,6 & 0,5 & 0,7 \\
\hline Total & 100,0 & 100,0 & 100,0 & 100,0 & 100,0 & 100,0 & 100,0 & 100,0 & 100,0 & 100,0 & 100,0 & 100,0 \\
\hline
\end{tabular}

Fonte: Sistema Nacional de Informações Tóxico-Farmacológicas, Centro de Informação Científica e Tecnológica, Fundação Oswaldo Cruz. 
As drogas de abuso, agente tóxico bastante explorado na literatura quando se trata de adolescentes e pré-adolescentes, contribuíram com percentuais inferiores a $7 \%$ em todas as regiões, representando o quinto principal agente tóxico apenas na Região Sudeste, para os adolescentes de 15 a 19 anos (Tabela 1).

Contudo, ao analisarmos a participação percentual dessas duas faixas etárias para cada um dos agentes tóxicos, verificamos que a segunda maior prevalência entre as intoxicações por drogas de abuso e a terceira entre as intoxicações por medicamentos e raticidas ocorrem em adolescentes. Já os pré-adolescentes não constituem uma das três principais faixas etárias para qualquer um dos agentes tóxicos considerados pelo SINITOX (Tabela 2).

Com relação aos coeficientes de incidência das intoxicações, calculados por agente tóxico e faixa etária, verificamos que os adolescentes apresentam o maior valor em relação às demais faixas etárias para as drogas de abuso, o segundo maior valor para os medicamentos e raticidas e o terceiro maior valor para agrotóxicos de uso doméstico e cosméticos. Os pré-adolescentes apresentam apenas o terceiro maior valor para plantas (Tabela 2).

Dos 35 óbitos por intoxicação de pré-adolescentes registrados no país pelo SINITOX, $54,3 \%$ foram acidentais, $54,3 \%$ acometeram o sexo masculino e $31,4 \%$ foram causados por animais peçonhentos. Contudo, observa-se maior prevalência do sexo feminino nos óbitos de intoxicação por medicamentos, agrotóxicos de uso agrícola e raticidas (Tabela 3 ).

Dos 98 óbitos por intoxicação de adolescentes registrados, $72,4 \%$ foram resultado de suicídio, $54,1 \%$ acometeram o sexo feminino e $38,8 \%$ foram causados por agrotóxicos de uso agrícola. No entanto, observa-se maior participação do sexo masculino nos óbitos de intoxicação por agrotóxicos de uso agrícola e animais peçonhentos (Tabela 3 ).

Considerando a totalidade dos casos registrados pelo SINITOX, verifica-se que o acidente foi a principal circunstância envolvida nos casos de intoxicação $(60,4 \%)$, seguido pela tentativa de suicídio (20\%). Ao analisar a distribuição percentual das circunstâncias definidas pelo SINITOX para cada um dos agentes tóxicos, verifica-se que para os agrotóxicos de uso agrícola e raticidas há uma inversão, a tentativa de suicídio se torna a principal circunstância (37\% e $57,3 \%$, respectivamente), seguida do acidente $(31,5 \%$ e $37,1 \%$, respectivamente). Com relação às drogas de abuso, praticamente não existem diferenças entre as proporções referentes à tentativa de suicídio $(6,1 \%)$ e aciden- te $(5,9 \%)$, sendo que a principal circunstância é o abuso (80,5\%). Os produtos químicos industriais, que compreendem os solventes ou inalantes, apresentam a circunstância "abuso" como a quarta mais freqüente $(1,8 \%)$, precedida por "acidente" (68\%), "ocupacional" (19,9\%) e "tentativa de suicídio" (7\%).

Entre as 43.648 tentativas de suicídio registradas pelo SINITOX, os medicamentos responderam por $54,8 \%$, seguidos pelos raticidas $(15,6 \%)$ e agrotóxicos de uso agrícola $(12,9 \%)$. Com relação aos 5.788 casos relacionados à circunstância "abuso", 74,1\% fizeram uso de drogas, $16,2 \%$ de medicamentos e $4,6 \%$ de produtos químicos industriais.

Com relação aos 218.692 casos de intoxicação registrados pelo SINITOX, a participação do sexo masculino $(50,2 \%)$ é pouco superior a do sexo feminino $(48,5 \%)$. Contudo, ao analisarmos cada agente tóxico separadamente, verificamos maior participação do sexo masculino nas intoxicações por agrotóxicos de uso agrícola, produtos veterinários, produtos químicos industriais, metais, drogas de abuso, animais peçonhentos e outros, enquanto que nas intoxicações por medicamentos, raticidas e alimentos temos maior participação do sexo feminino.

Já com relação aos 1.233 óbitos por intoxicação registrados pelo SINITOX, 63,1\% ocorreram entre pessoas do sexo masculino contra $36,3 \%$ entre pessoas do sexo feminino. Conseqüentemente, a letalidade por intoxicações para o sexo masculino é maior do que a do sexo feminino, $0,7 \%$ contra $0,4 \%$.

\section{Discussão}

Os pré-adolescentes constituem uma faixa etária em que a questão das intoxicações é pouco relevante ou, ao menos, discutida com menor freqüência. Uma explicação para este achado pode ser o fato desses jovens não serem mais tão crianças para experimentarem as conseqüências das intoxicações acidentais infantis e, por outro lado, não possuírem autonomia suficiente para ter acesso ao consumo de drogas e, mesmo os que as consome, ainda não tiveram tempo suficiente para que este consumo gere danos observáveis, passíveis de registro, limitando-se muitas vezes à experimentação e ao uso eventual 5,15,16. Além disso, parecem ainda não encarar o suicídio como uma possível solução para seus problemas existenciais, uma vez que para esses pré-adolescentes, como também para as crianças menores de dez anos e adultos com oitenta ou mais anos, o suicídio não constitui a principal circunstância dos óbi- 
Distribuição dos casos de intoxicação humana e seus respectivos coeficientes de incidência por 100 mil habitantes, segundo agente tóxico e faixa etária. Brasil, 1999 a 2001.

\begin{tabular}{|c|c|c|c|c|c|c|c|c|c|c|c|c|c|}
\hline \multirow[t]{2}{*}{ Agentes tóxicos } & \multicolumn{13}{|c|}{ Faixas etárias (anos) } \\
\hline & $<5$ & $5-9$ & $10-14$ & $15-19$ & $20-29$ & $30-39$ & $40-49$ & $50-59$ & $60-69$ & $70-79$ & 80 e + & Ignorado & Total \\
\hline \multicolumn{14}{|l|}{ Medicamentos } \\
\hline Casos & 23.239 & 4.294 & 3.232 & 7.033 & 10.224 & 6.396 & 3.828 & 1.585 & 732 & 396 & 188 & \multirow[t]{2}{*}{1.390} & 62.537 \\
\hline Coeficiente & 55,98 & 10,19 & 7,32 & 15,32 & 13,11 & 9,56 & 7,44 & 4,75 & 3,35 & 3,26 & 3,87 & & 14,14 \\
\hline \multicolumn{14}{|l|}{$\begin{array}{l}\text { Agrotóxicos de } \\
\text { uso agrícola }\end{array}$} \\
\hline Casos & 1.527 & 388 & 463 & 1.476 & 3.607 & 3.052 & 2.136 & 1.136 & 586 & 243 & 44 & \multirow[t]{2}{*}{527} & 15.185 \\
\hline Coeficiente & 3,68 & 0,92 & 1,05 & 3,21 & 4,63 & 4,56 & 4,15 & 3,40 & 2,68 & 2,00 & 0,91 & & 3,43 \\
\hline \multicolumn{14}{|l|}{$\begin{array}{l}\text { Agrotóxicos de } \\
\text { uso doméstico }\end{array}$} \\
\hline Casos & 2.496 & 394 & 337 & 730 & 1.536 & 992 & 644 & 309 & 126 & 101 & 29 & \multirow[t]{2}{*}{245} & 7.939 \\
\hline Coeficiente & 6,01 & 0,94 & 0,76 & 1,59 & 1,97 & 1,48 & 1,25 & 0,93 & 0,58 & 0,83 & 0,60 & & 1,80 \\
\hline \multicolumn{14}{|c|}{ Produtos veterinários } \\
\hline Casos & 538 & 87 & 96 & 189 & 353 & 322 & 201 & 118 & 81 & 21 & 4 & \multirow[t]{2}{*}{90} & 2.100 \\
\hline Coeficiente & 1,30 & 0,21 & 0,22 & 0,41 & 0,45 & 0,48 & 0,39 & 0,35 & 0,37 & 0,17 & 0,08 & & 0,47 \\
\hline \multicolumn{14}{|l|}{ Raticidas } \\
\hline Casos & 3.317 & 366 & 609 & 1.802 & 2.645 & 1.482 & 862 & 347 & 132 & 69 & 25 & \multirow[t]{2}{*}{247} & 11.903 \\
\hline Coeficiente & 7,99 & 0,87 & 1,38 & 3,92 & 3,39 & 2,22 & 1,67 & 1,04 & 0,60 & 0,57 & 0,51 & & 2,69 \\
\hline \multicolumn{14}{|l|}{ Domissanitários } \\
\hline Casos & 10.704 & 1.223 & 660 & 1.138 & 2.062 & 1.456 & 958 & 494 & 252 & 129 & 66 & \multirow[t]{2}{*}{565} & 19.707 \\
\hline Coeficiente & 25,79 & 2,90 & 1,50 & 2,48 & 2,64 & 2,18 & 1,86 & 1,48 & 1,15 & 1,06 & 1,36 & & 4,46 \\
\hline \multicolumn{14}{|l|}{ Cosméticos } \\
\hline Casos & 1.558 & 129 & 67 & 92 & 112 & 69 & 45 & 35 & 16 & 7 & 5 & \multirow[t]{2}{*}{51} & 2.186 \\
\hline Coeficiente & 3,75 & 0,31 & 0,15 & 0,20 & 0,14 & 0,10 & 0,09 & 0,10 & 0,07 & 0,06 & 0,10 & & 0,49 \\
\hline $\begin{array}{l}\text { Produtos químice } \\
\text { industriais }\end{array}$ & & & & & & & & & & & & & \\
\hline Casos & 5.728 & 775 & 543 & 994 & 2.373 & 1.856 & 1.294 & 564 & 227 & 106 & 38 & 553 & 15.051 \\
\hline Coeficiente & 13,80 & 1,84 & 1,23 & 2,16 & 3,04 & 2,77 & 2,51 & 1,69 & 1,04 & 0,87 & 0,78 & & 3,40 \\
\hline Metais & & & & & & & & & & & & & \\
\hline Casos & 395 & 100 & 32 & 40 & 175 & 209 & 190 & 84 & 26 & 12 & 2 & 71 & 1.336 \\
\hline Coeficiente & 0,95 & 0,24 & 0,07 & 0,09 & 0,22 & 0,31 & 0,37 & 0,25 & 0,12 & 0,10 & 0,04 & & 0,30 \\
\hline Drogas de abuso & & & & & & & & & & & & & \\
\hline Casos & 170 & 85 & 302 & 1.024 & 1.706 & 1.000 & 526 & 191 & 67 & 15 & 4 & 236 & 5.326 \\
\hline Coeficiente & 0,41 & 0,20 & 0,68 & 2,23 & 2,19 & 1,49 & 1,02 & 0,57 & 0,31 & 0,12 & 0,08 & & 1,20 \\
\hline Plantas & & & & & & & & & & & & & \\
\hline Casos & 2.189 & 980 & 362 & 295 & 380 & 250 & 202 & 114 & 57 & 41 & 10 & 157 & 5.037 \\
\hline Coeficiente & 5,27 & 2,33 & 0,82 & 0,64 & 0,49 & 0,37 & 0,39 & 0,34 & 0,26 & 0,34 & 0,21 & & 1,14 \\
\hline Alimentos & & & & & & & & & & & & & \\
\hline Casos & 224 & 216 & 201 & 206 & 456 & 256 & 162 & 81 & 44 & 32 & 10 & 152 & 2.040 \\
\hline Coeficiente & 0,54 & 0,51 & 0,46 & 0,45 & 0,58 & 0,38 & 0,31 & 0,24 & 0,20 & 0,26 & 0,21 & & 0,46 \\
\hline Animais peçonhe & & & & & & & & & & & & & \\
\hline Casos & 3.504 & 4.092 & 4.244 & 4.551 & 9.250 & 8.064 & 6.253 & 4.036 & 2.541 & 1.155 & 354 & 1.082 & 49.126 \\
\hline Coeficiente & 8,44 & 9,71 & 9,62 & 9,91 & 11,86 & 12,05 & 12,15 & 12,09 & 11,63 & 9,52 & 7,29 & & 11,11 \\
\hline $\begin{array}{l}\text { Animais não- } \\
\text { peçonhentos }\end{array}$ & & & & & & & & & & & & & \\
\hline Casos & 1.230 & 1.018 & 914 & 1.109 & 2.324 & 1.878 & 1.460 & 857 & 515 & 241 & 60 & 555 & 12.161 \\
\hline Coeficiente & 2,96 & 2,42 & 2,07 & 2,42 & 2,98 & 2,81 & 2,84 & 2,57 & 2,36 & 1,99 & 1,24 & & 2,75 \\
\hline Desconhecido & & & & & & & & & & & & & \\
\hline Casos & 1.097 & 495 & 394 & 475 & 734 & 561 & 428 & 184 & 119 & 57 & 21 & 157 & 4.722 \\
\hline Coeficiente & 2,64 & 1,17 & 0,89 & 1,03 & 0,94 & 0,84 & 0,83 & 0,55 & 0,54 & 0,47 & 0,43 & & 1,07 \\
\hline Outro & & & & & & & & & & & & & \\
\hline Casos & 860 & 223 & 135 & 156 & 286 & 248 & 143 & 85 & 50 & 23 & 4 & 123 & 2.336 \\
\hline Coeficiente & 2,07 & 0,53 & 0,31 & 0,34 & 0,37 & 0,37 & 0,28 & 0,25 & 0,23 & 0,19 & 0,08 & & 0,53 \\
\hline Total & & & & & & & & & & & & & \\
\hline Casos & 123 & 14.865 & 12.591 & 21.310 & 38.223 & 28.091 & 19.332 & 10.220 & 5.571 & 2.648 & 864 & 6.201 & 218.692 \\
\hline Coeficiente & 141,60 & 35,28 & 28,53 & 46,41 & 49,02 & 41,99 & 37,56 & 30,62 & 25,50 & 21,82 & 17,80 & & 49,45 \\
\hline
\end{tabular}

Fonte: Sistema Nacional de Informações Tóxico-Farmacológicas, Centro de Informação Científica e Tecnológica, Fundação Oswaldo Cruz. 
Tabela 3

Distribuição de óbitos por intoxicação humana em pré-adolescentes de 10 a 14 anos e em adolescentes de 15 a 19 anos, segundo agente tóxico, circunstância e sexo. Brasil, 1999 a 2001.

\begin{tabular}{|c|c|c|c|c|c|c|c|c|c|c|c|c|c|c|c|c|c|c|c|c|c|c|c|c|}
\hline \multirow[t]{3}{*}{ Agentes tóxicos } & \multicolumn{12}{|c|}{ Pré-adolescentes de 10 a 14 anos } & \multicolumn{12}{|c|}{ Adolescentes de 15 a 19 anos } \\
\hline & \multicolumn{2}{|c|}{ Acidente } & \multicolumn{2}{|c|}{ Abuso } & \multicolumn{2}{|c|}{ Suicídio } & \multicolumn{2}{|c|}{$\begin{array}{l}\text { Uso te- } \\
\text { rapêutico }\end{array}$} & \multicolumn{2}{|c|}{ Ignorada } & \multicolumn{2}{|c|}{ Total } & \multicolumn{2}{|c|}{ Acidente } & \multicolumn{2}{|c|}{ Abuso } & \multicolumn{2}{|c|}{ Suicídio } & \multicolumn{4}{|c|}{$\begin{array}{l}\text { Tentativa Ignorada } \\
\text { de aborto }\end{array}$} & \multicolumn{2}{|c|}{ Total } \\
\hline & M & $\mathrm{F}$ & M & $\mathrm{F}$ & M & $\mathrm{F}$ & M & $\mathrm{F}$ & M & $\mathrm{F}$ & M & $\mathrm{F}$ & M & $\mathrm{F}$ & M & $\mathrm{F}$ & M & $\mathrm{F}$ & M & $\mathrm{F}$ & M & $\mathrm{F}$ & M & $\mathrm{F}$ \\
\hline Medicamentos & - & 1 & - & - & 1 & 1 & - & 1 & - & - & 1 & 3 & - & 1 & - & - & 4 & 12 & - & 1 & 1 & 1 & 5 & 15 \\
\hline $\begin{array}{l}\text { Agrotóxicos de } \\
\text { uso agrícola }\end{array}$ & 1 & - & - & - & - & 4 & - & - & - & - & 1 & 4 & 3 & - & - & - & 17 & 14 & - & - & 3 & 1 & 23 & 15 \\
\hline $\begin{array}{l}\text { Agrotóxicos de } \\
\text { uso doméstico }\end{array}$ & - & - & - & - & - & - & - & - & - & - & - & - & - & - & - & - & - & - & - & - & - & - & - & - \\
\hline Produtos veterinários & - & - & - & - & - & - & - & - & - & - & - & - & 1 & - & - & - & 1 & - & - & - & - & - & 2 & - \\
\hline Raticidas & - & 1 & - & - & 1 & 2 & - & - & 1 & - & 2 & 3 & - & - & - & - & 4 & 14 & - & - & - & - & 4 & 14 \\
\hline Domissanitários & - & 1 & - & - & - & - & - & - & - & - & - & 1 & - & - & - & - & - & 1 & - & - & - & - & - & 1 \\
\hline Cosméticos & - & - & - & - & - & - & - & - & - & - & - & - & - & - & - & - & - & - & - & - & - & - & - & - \\
\hline $\begin{array}{l}\text { Produtos químicos } \\
\text { industriais }\end{array}$ & 1 & 1 & - & - & - & - & - & - & - & - & 1 & 1 & 1 & - & - & - & 2 & 2 & - & - & - & - & 3 & 2 \\
\hline Metais & - & - & - & - & - & - & - & - & - & - & - & - & - & - & - & - & - & - & - & - & - & - & - & - \\
\hline Drogas de abuso & - & - & 1 & - & - & - & - & - & 1 & - & 2 & - & - & - & 2 & - & - & - & - & - & - & - & 2 & - \\
\hline Plantas & - & - & - & - & - & - & - & - & - & - & - & - & 1 & - & - & - & - & - & - & 1 & - & 1 & 1 & 2 \\
\hline Alimentos & - & - & - & - & - & - & - & - & - & - & - & - & - & - & - & - & - & - & - & - & - & - & - & - \\
\hline Animais peçonhentos & 9 & 2 & - & - & - & - & - & - & - & - & 9 & 2 & 2 & - & - & - & - & - & - & - & 1 & - & 3 & - \\
\hline $\begin{array}{l}\text { Animais não- } \\
\text { peçonhentos }\end{array}$ & - & - & - & - & - & - & - & - & - & - & - & - & - & - & - & - & - & - & - & - & - & - & - & - \\
\hline Desconhecido & 1 & - & - & - & - & - & - & - & - & 1 & 1 & 1 & - & 2 & - & - & - & - & - & - & 2 & 2 & 2 & 4 \\
\hline Outro & 1 & - & - & - & - & - & - & - & 1 & 1 & 2 & 1 & - & - & - & - & - & - & - & - & - & - & - & - \\
\hline Total & 13 & 6 & 1 & - & 2 & 7 & - & 1 & 3 & 2 & 19 & 16 & 8 & 3 & 2 & - & 28 & 43 & - & 2 & 7 & 5 & 45 & 53 \\
\hline
\end{tabular}

Fonte: Sistema Nacional de Informações Tóxico-Farmacológicas, Centro de Informação Científica e Tecnológica, Fundação Oswaldo Cruz.

tos por intoxicação registrados pelo SINITOX. Estes pré-adolescentes não se comportam como as crianças menores de dez anos e nem como os adolescentes de 15 a 19 anos e adultos jovens de 20 a 29 anos, pois para eles os medicamentos não constituem o principal agente tóxico.

As intoxicações por drogas de abuso registradas pelo SINITOX apresentam participações porcentuais inferiores a $5 \%$ para todas as faixas etárias, sendo os maiores valores encontrados entre os adolescentes, seguidos pelos adultos jovens. Entre as intoxicações por drogas de abuso há mais casos de adultos jovens, seguidos dos de adolescentes. Contudo, ao considerarmos as populações de cada faixa etária, verificamos que o coeficiente de incidência de intoxicações por drogas de abuso dos adolescentes $(2,23)$ é um pouco superior ao dos adultos jovens $(2,19)$, levando a um risco relativo de 1,02 , o que significa que a chance de um adolescente vir a se intoxicar por drogas de abuso é praticamente a mesma de um adulto jovem. Já o risco relativo de um adolescente vir a se in- toxicar por drogas de abuso em relação a um pré-adolescente é de 3,26, o que está de acordo com o aumento do consumo "regular" de álcool a partir dos 15 anos 5,16.

O “chumbinho", agrotóxico de uso agrícola do grupo dos carbamatos, desviado clandestinamente para as cidades para ser vendido pelo comércio ambulante como raticida, pode ser classificado pelos Centros de Informação e Assistência Toxicológica tanto como agrotóxico de uso agrícola, se for considerado sua característica original, tanto como raticida, se for considerado seu uso inadequado para matar ratos nas cidades. Com base nos dados apresentados por Schanuel et al. 17, sobre intoxicações por "chumbinho" registradas no período de janeiro de 1997 a junho de 1998 pelos dois centros de controle de intoxicações existentes no Estado do Rio de Janeiro (CCIn do Hospital Universitário Antônio Pedro e CCI do Hospital Universitário Clementino Fraga Filho), verificamos que este tipo de intoxicação ocorre com uma freqüência um pouco maior para o sexo feminino, $51,2 \%$ contra $46,2 \%$, atingem principalmente 
as faixas etárias de 21 a 40 anos e de 16 a 20 anos, e a circunstância "tentativa de suicídio" é apontada na maioria dessas ocorrências. Esta caracterização do "chumbinho" pode explicar o fato das intoxicações por agrotóxicos de uso agrícola e por raticidas apresentarem como principal circunstância a tentativa de suicídio.

As intoxicações por produtos químicos industriais, que tiveram como circunstância o abuso, poderiam traduzir as intoxicações por inalantes ou solventes. Para os 5.788 casos de intoxicação por abuso, considerando todas as faixas etárias, $74,1 \%$ fizeram uso de drogas, $16,2 \%$ de medicamentos e $4,6 \%$ de produtos químicos industriais. Esta distribuição está de acordo com Scivoletto et al. 15, que analisando 21 pacientes com idades entre 11 e 17 anos, que demandaram tratamento em um serviço público terciário para dependência de álcool e/ou drogas em regime ambulatorial, concluíram que os usuários de solventes buscam raramente tratamento, em relação à sua prevalência elevada na população de adolescentes.

Com relação ao consumo de drogas por adolescentes, Galduróz et al. 18, estudando o uso de drogas entre estudantes de 1o e 2 o graus em dez capitais brasileiras, verificaram uma prevalência mais elevada de uso na vida de solventes, nos quatro levantamentos realizados (1987, 1989, 1993 e 1997), excluindo-se o tabaco e o álcool. Estes resultados foram também encontrados por Muza et al. 19, Carlini-Cotrim et al. 20, Godoi et al. 21 e Bucher et al. 22, em seus estudos com estudantes de escolas públicas e privadas.

No entanto, Carlini et al. 23 , realizando pesquisa domiciliar sobre consumo de drogas, verificaram maior prevalência de uso na vida de maconha, excluídos o álcool e o tabaco, o que pode sugerir que os adolescentes que estão fora do sistema escolar fazem uso mais freqüente dessa droga. Este achado pode estar relacionado ao fato do consumo de maconha contribuir para o afastamento do adolescente do meio escolar, uma vez que o uso dessa droga pode comprometer a memória e o rendimento intelectual e, em especial, estigmatizar e criminalizar o adolescente, o que acaba culminando com sua expulsão da escola. Este achado pode também estar relacionado ao fato desses adolescentes já estarem fora do ambiente escolar e assim constituírem um alvo mais fácil para a experimentação ou mesmo para o consumo dessa droga. Além disso, deveríamos também considerar que o uso dos solventes ocorre com maior freqüência de forma sazonal ou mesmo ocasional, podendo muitas vezes ser consumido dentro da escola sem cau- sar maiores alardes, implicando assim, menor evasão escolar.

A maior prevalência do sexo masculino nas intoxicações por drogas de abuso e do sexo feminino nas intoxicações por medicamentos estão de acordo com a literatura, que registra o maior consumo de drogas por adolescentes do sexo masculino e de medicamentos por adolescentes do sexo feminino 17,19,24.

$\mathrm{Na}$ impossibilidade de analisarmos as tentativas de suicídio por sexo, lançamos mão de artigos como os de Cassorla 25, Fernandes et al. 26, Teixeira et al. 27, Rapeli et al. 28, Marcondes Filho et al. 29 e Feijó et al. 30, que afirmam que suicídio e tentativa de suicídio são, do ponto de vista epidemiológico, fenômenos que ocorrem em populações diferentes. Os homens são mais suscetíveis ao suicídio, tentam o suicídio menos freqüentemente, mas com métodos mais eficazes e, portanto, apresentam êxito em maior número de vezes, e as mulheres tentam mais freqüentemente o suicídio e têm menos êxito por usarem drogas menos tóxicas. Mello-Jorge et al. 31, verificaram que os coeficientes de mortalidade por suicídio são sempre maiores no sexo masculino, contrariamente às tentativas de suicídio, mais numerosas entre as mulheres.

Garrido-Romero et al. 32 identificaram a tentativa de suicídio como a emergência psiquiátrica mais freqüente nos adolescentes, sendo a idade média dos pacientes admitidos com intoxicação voluntária em serviço de emergência para crianças e adolescentes de 15,6 anos, dos quais $87 \%$ eram do sexo feminino.

Lebrão et al. 33 , ao analisarem a morbidade hospitalar por lesões e envenenamentos, verificam maior prevalência do sexo masculino sobre o feminino tanto para os pré-adolescentes quanto para os adolescentes, o que é também verificado por Travassos et al. ${ }^{34}$ para os jovens de 15 a 24 anos. Com relação às internações por envenenamentos apenas, ocorre exatamente o inverso, com uma maior prevalência do sexo feminino às custas das intoxicações por drogas e medicamentos 33. Como os dados do SINITOX apontam para uma maior prevalência do sexo masculino, podemos pensar que os casos que exigiram internação estão mais freqüentemente relacionados com os agentes tóxicos em que são observadas as maiores prevalências do sexo feminino como os medicamentos, raticidas e alimentos. Além disso, como já discutido anteriormente, as tentativas de suicídio são mais freqüentes entre mulheres, o que pode implicar um número maior de internações.

Minayo et al. 35, também analisando a morbidade hospitalar por lesões e envenenamentos, observam que estas constituem a segunda 
causa de internação entre jovens, de 10 a 19 anos, e entre adultos jovens, de 20 a 29 anos. Dentre as principais causas de internações por lesões e envenenamentos destacam-se os traumatismos, seguidos das queimaduras e das intoxicações não medicamentosas.

Mello-Jorge 36, analisando dados de óbitos por todas as causas de jovens de 15 a 24 anos, verificou uma clara predominância do sexo masculino. Os coeficientes de mortalidade foram mais elevados para jovens de 20 a 24 anos do sexo masculino, seguidos pelos adolescentes de 10 a 19 anos do mesmo sexo. Entre os jovens de 15 a 24 anos do sexo feminino, os valores são também um pouco mais elevados no grupo de 20 a 24 anos, em relação ao de 15 a 19. Mais da metade das mortes de adolescentes e de jovens de 20 a 24 anos ocorreram por causas externas, sendo estes óbitos preponderantemente do sexo masculino.

Resumo

Este trabalho analisa o perfil das intoxicações em adolescentes valendo-se da base de dados do Sistema Nacional de Informações Tóxico-Farmacológicas (SINITOX), composta de casos e óbitos de intoxicação humana registrados pelos Centros de Informação e Assistência Toxicológica, para o período de 1999 a 2001. Com relação aos casos, animais peçonhentos $(33,7 \%) e$ medicamentos $(25,7 \%)$ constituem os principais agentes tóxicos para os pré-adolescentes de 10 a 14 anos, enquanto para os adolescentes de 15 a 19 anos temos uma inversão das posições, com os medicamentos (33\%), sendo seguidos pelos animais peçonhentos (21,4\%). Dos 35 óbitos de pré-adolescentes registrados, $54,3 \%$ foram resultado de acidente, 54,3\% acometeram jovens do sexo masculino e $31,4 \%$ foram causados por animais peçonhentos. Dos 98 óbitos de adolescentes, $72,4 \%$ foram resultado de suicídio, sendo $54,1 \%$ praticados por jovens do sexo feminino e 38,8\% foram causados por agrotóxicos de uso agrícola.

Envenenamento; Substâncias Tóxicas; Adolescente

\section{Agradecimentos}

À Maria de Lourdes de Oliveira Justino e às alunas do Programa de Vocação Científica da FIOCRUZ, Camila Mitrano da Costa e Silva Raposo e Rachel Galdino Sarmento, pela participação na pesquisa bibliográfica e coleta das referências.

\section{Conclusões}

Os pré-adolescentes e os adolescentes apresentam comportamentos diferentes frente às intoxicações. As intoxicações em pré-adolescentes são menos freqüentes e podem ser resumidas a acidentes causados por animais peçonhentos, que acometem mais indivíduos do sexo masculino, enquanto as intoxicações em adolescentes são mais freqüentes e estão mais relacionadas às tentativas de suicídio com o uso de medicamentos, praticadas por indivíduos do sexo feminino.

Com exceção das intoxicações por plantas, as demais apresentam maior impacto para os adolescentes do que para os pré-adolescentes, em especial as causadas por drogas de abuso, agrotóxicos de uso agrícola e raticidas, sendo a chance de um adolescente apresentar intoxicação por cada um desses agentes tóxicos superior a três vezes a chance do mesmo ocorrer com um préadolescente. Intoxicações por medicamentos e por agrotóxicos de uso doméstico também se destacam para os adolescentes em relação aos pré-adolescentes, com mais do dobro de chance de apresentar cada uma dessas intoxicações.

\section{Referências}

1. Instituto Brasileiro de Geografia e Estatística. Censo Demográfico 2000. http://www.ibge.gov.br/ home/estatistica/populacao/censo2000 (acessado em 28/Out/2004).

2. Houaiss A, editor. Enciclopédia mirador internacional. São Paulo: Encyclopaedia Britannica do Brasil Publicações Ltda.; 1975.

3. Grande Enciclopédia Larousse Cultural. São Paulo: Nova Cultural Ltda.; 1998.

4. Becker D. O que é adolescência. São Paulo: Editora Brasiliense; 2003.

5. Zago JA. Álcool e adolescência. http://www.adroga.casadia.org/alcoolismo/alcool_e_adolescencia.htm (acessado em 28/Out/2004).

6. Marques MB, Bortoletto ME, Bezerra MCC, Santana RAL. Avaliação da rede brasileira de Centros de Controle de Intoxicações e Envenenamentos CCIEs. Cad Saúde Pública 1995; 11:560-78.

7. Bortoletto ME, Bochner R. Impacto dos medicamentos nas intoxicações humanas no Brasil. Cad Saúde Pública 1999; 15:859-69.

8. Bochner R, Struchiner CJ. Acidentes por animais peçonhentos e sistemas nacionais de informação. Cad Saúde Pública 2002; 18:735-46.

9. Sistema Nacional de Informações Tóxico-Farmacológicas, Centro de Informação Científica e Tecnológica, Fundação Oswaldo Cruz. Estatística anual de casos de intoxicação e envenenamento. Brasil, 1999. Rio de Janeiro: Sistema Nacional de Informações Tóxico-Farmacológicas, Centro de 
Informação Científica e Tecnológica, Fundação Oswaldo Cruz; 2000.

10. Sistema Nacional de Informações Tóxico-Farmacológicas, Centro de Informação Científica e Tecnológica, Fundação Oswaldo Cruz. Estatística anual de casos de intoxicação e envenenamento. Brasil, 2000. Rio de Janeiro: Sistema Nacional de Informações Tóxico-Farmacológicas, Centro de Informação Científica e Tecnológica, Fundação Oswaldo Cruz; 2002.

11. Sistema Nacional de Informações Tóxico-Farmacológicas, Centro de Informação Científica e Tecnológica, Fundação Oswaldo Cruz. Estatística anual de casos de intoxicação e envenenamento. Brasil, 2001. http://www.fiocruz.br/sinitox (acessado em 28/Out/2004).

12. Campos DMS. Psicologia da adolescência. Petrópolis: Editora Vozes; 2002.

13. Brasil. Estatuto da Criança e do Adolescente. Brasília: Secretaria de Fiscalização do Trabalho, Ministério do Trabalho; 1998.

14. Organización Panamericana de la Salud/Organización Mundial de la Salud. La salud del adolescente y el joven en las Américas. Washington DC: Organización Panamericana de la Salud/Organización Mundial de la Salud; 1985. (Publicación Científica, 489).

15. Scivoletto S, Henriques Jr. SG, Andrade AG. Uso de drogas por adolescentes que buscam atendimento ambulatorial: comparação entre "crack" e outras drogas ilícitas - um estudo piloto. Rev ABP-APAL 1997; 19:7-17.

16. Carlini BH, Pires MLN, Fernandes R, Masur J. O consumo de bebidas alcoólicas entre estudantes de primeiro grau na Cidade de São Paulo. J Bras Psiquiatr 1986; 35:279-85.

17. Schanuel ALR, Oliveira RMR, Levigard YE. Intoxicações por chumbinho: uma epidemia silenciosa [Monografia de Especialização]. Rio de Janeiro: Escola Nacional de Saúde Pública, Fundação Oswaldo Cruz; 1998.

18. Galduróz JCF, Noto NA, Carlini EA. IV levantamento sobre o uso de drogas entre estudantes de 1o e 2o graus em 10 capitais brasileiras-1997. São Paulo: Centro Brasileiro de Informações sobre Drogas Psicotrópicas, Universidade Federal de São Paulo; 1997.

19. Muza GM, Bettiol H, Muccilo G, Barbieri MA. Consumo de substâncias psicoativas por adolescentes escolares de Ribeirão Preto, SP (Brasil). I Prevalência do consumo por sexo, idade e tipo de substância. Rev Saúde Pública 1997; 31:21-9.

20. Carlini-Cotrim B, Carlini EA. O consumo de solventes e outras drogas em crianças e adolescentes de baixa renda na Cidade de São Paulo. Parte I: estudantes de primeiro e segundo graus da rede estadual. Rev ABP-APAL 1987; 9:49-58.

21. Godoi AMM, Muza GM, Costa MP, Gama MLT. Consumo de substâncias psicoativas por escolares da rede privada do Distrito Federal. Rev Saúde Pública 1991; 25:150-6.

22. Bucher R, Totugui ML. Conhecimento e uso de drogas entre alunos de Brasília. Psicol Teor Pesqui 1987; 3:178-94
23. Carlini EA, Galduróz JCF, Noto AR, Nappo SA. I levantamento domiciliar sobre o uso de drogas psicotrópicas no Brasil: estudo envolvendo as 107 maiores cidades do país - 2001. São Paulo: Centro Brasileiro de Informações sobre Drogas Psicotrópicas, Universidade Federal de São Paulo; 2002.

24. Tavares BF, Béria JU, Lima MS. Prevalência do uso de drogas e desempenho escolar entre adolescentes. Rev Saúde Pública 2001; 35:150-8.

25. Cassorla RMS. Jovens que tentam suicídio: características demográficas e sociais. J Bras Psiquiatr 1984; 33:3-12.

26. Fernandes IMM, Sato AK, Pires Jr. HS, Serafim SR, Castro RP, Catelan GM, et al. Levantamento de 302 Tentativas de Suicídio do Centro de Assistência Toxicológica do Hospital de Base de 01/92 a 02/93. J Bras Med 1995; 68:209-15.

27. Teixeira AMF, Luis MAV. Suicídio, lesões e envenenamento em adolescentes: um estudo epidemiológico. Rev Latinoam Enfermagem 1997; 5:31-6.

28. Rapeli CB, Botega NJ. Tentativas de suicídio envolvendo risco de vida: internações em um hospital geral. J Bras Psiquiatr 1998; 47:157-62.

29. Marcondes Filho W, Mezzaroba L, Turini CA, Koike A, Motomatsu Jr. A, Shibayama EEM, et al. Tentativas de suicídio por substâncias químicas na adolescência e juventude. Adolesc Latinoam 2002; 3:0-0.

30. Feijó RB, Raupp APG, John AB. Eventos estressores de vida e sua relação com tentativas de suicídio em adolescentes. J Bras Psiquiatr 1999; 8:151-7.

31. Mello-Jorge MH, Gawryszewski VP, Latorre MRDO. I - análise dos dados de mortalidade. Rev Saúde Pública 1997; 31 Suppl 4:5-25.

32. Garrido-Romero R, García-García JJ, CarballoRuano E, Luaces-Cubells C, Alda-Díez JA. Intoxicaciones voluntárias como intento de suicidio. An Esp Pediatr 2000; 53:213-6.

33. Lebrão ML, Mello-Jorge MH, Laurenti R. II - Morbidade hospitalar por lesões e envenenamentos. Rev Saúde Pública 1997; 31 Suppl 4:26-37.

34. Travassos C, Lebrão ML. Morbidade hospitalar nos jovens. In: Berquó E, organizador. Jovens acontecendo na trilha das políticas públicas. Brasília: Comissão Nacional de População e Desenvolvimento; 1998. p. 165-96.

35. Minayo MCS, Souza ER, Silva CMFP, organizadores. Análise da morbidade hospitalar por lesões e envenenamentos no Brasil em 1999. Rio de Janeiro: Centro Latino-americano de Estudos de Violência e Saúde Jorge Careli, Fundação Oswaldo Cruz; 1991.

36. Mello-Jorge MH. Como morrem nossos jovens. In: Berquó E, organizador. Jovens acontecendo na trilha das políticas públicas. Brasília: Comissão Nacional de População e Desenvolvimento; 1998. p. 209-89.

Recebido em 06/Dez/2004

Versão final reapresentada em 26/Set/2005

Aprovado em 27/Set/2005 